\title{
Total Resection of Cervical Ventral Intramedullary Cavernous Hemangiomas with an Anterior Corpectomy
}

\author{
Narihito Nagoshi $^{1)}$, Ken Ishii ${ }^{12) 33}$, Kaori Kameyama ${ }^{4)}$, Osahiko Tsuji ${ }^{1)}$, Eijiro Okada ${ }^{1)}$, Nobuyuki Fujita ${ }^{1)}$, Mitsuru Yagi ${ }^{1)}$, \\ Morio Matsumoto $^{1)}$, Masaya Nakamura ${ }^{1)}$ and Kota Watanabe ${ }^{1)}$ \\ 1) Department of Orthopaedic Surgery, Keio University School of Medicine, Tokyo, Japan \\ 2) Spine and Spinal Cord Center, International University of Health and Welfare, Mita Hospital, Tokyo, Japan \\ 3) Department of Orthopaedic Surgery, School of Medicine, International University of Health and Welfare, Chiba, Japan \\ 4) Division of Diagnostic Pathology, Keio University School of Medicine, Tokyo, Japan
}

\begin{abstract}
:
Introduction: Intramedullary lesions and tumors are generally accessed by a posterior approach. However, if the lesion is located on the ventral side of the spinal cord, a posterior resection with myelotomy poses technical difficulties. We report two cases of complete resection of a cervical ventral intramedullary cavernous hemangioma using an anterior approach.

Case Report: Two cases of intramedullary cavernous hemangioma located on the ventral side of the spinal cord were successfully treated by total resection with anterior cervical corpectomy followed by anterior spinal fusion with an autologous bone strut from the iliac crest. In both cases, the postoperative course was uneventful, and there was no neurological deficit. Bony fusion was achieved, and there was no recurrence or complication during a follow-up period of at least two years.
\end{abstract}

Conclusions: Here, we describe an anterior approach for total resection of cavernous hemangiomas on the ventral side of the cervical spinal cord. Outcomes were stable two years after the operations. Although the method should be assessed with more patients and a longer follow-up time, this anterior approach may be useful for the radical resection of a vascular malformation or tumor.

Keywords:

Cavernous hemangiomas, Ventral side of spinal cord, Intramedullary lesions, Anterior approach, Surgical outcomes

Spine Surg Relat Res 2018; 2(4): 331-334

dx.doi.org/10.22603/ssrr.2017-0088

\section{Introduction}

Intramedullary spinal cord cavernous hemangioma $(\mathrm{CH})$ accounts for $5 \%-12 \%$ of all spinal cord vascular lesions ${ }^{1}$. The clinical course of symptomatic $\mathrm{CH}$ is aggressive, with a high bleeding rate, so surgical resection is recommended ${ }^{2}$. Myelotomy through a posterior or posterolateral approach generally is conducted for the tumor resection ${ }^{1)}$. However, a lesion on the ventral side of the spinal cord is technically difficult to access by the posterior approach. Here we report the technique and surgical outcomes of tumor resection followed by anterior cervical corpectomy and fusion (ACCF).

\section{Case 1}

A 47-year-old male presented with sensory disturbance of the left upper extremity. T2-weighted magnetic resonance imaging (MRI) showed a heterogeneous intensity mass with hemosiderin deposition in the ventral region of the spinal cord at the C6 level (Fig. 1). The diagnosis was suspected to be $\mathrm{CH}$, and surgical resection was performed (Video, Supplemental Digital Content 1). C6 corpectomy was performed over a wide region, with partial resection of the $\mathrm{C} 5$ and $\mathrm{C} 7$ vertebral bodies, especially at the dorsal side of the vertebrae, to ease the subsequent procedure. The Luschka's joints were completely preserved. The obtainment of a sufficient 




Figure 1. Preoperative T2-weighted MRI at sagittal (left) and axial (right) sections. Note the heterogeneous intensity mass with hemosiderin deposition in the ventral region of the spinal cord at the C6 level (arrowheads).
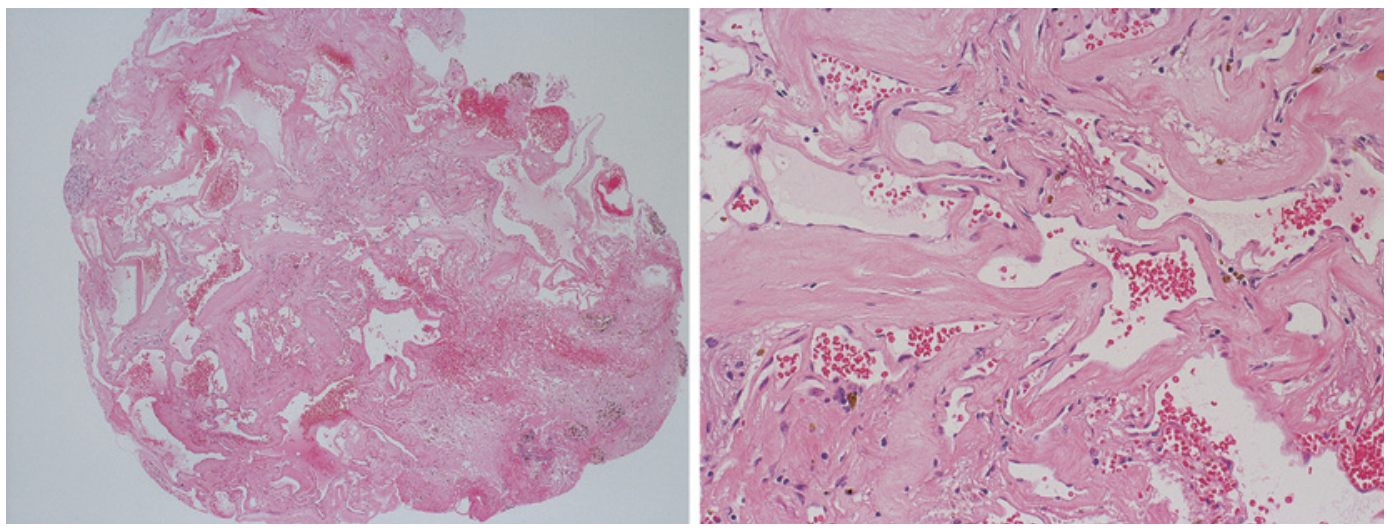

Figure 2. Hematoxylin-eosin staining of resected lesions at lower (left) and higher (right) magnifications. Dilated thin-walled vessels with flattened endothelial cells are prominent.

operating field for tumor resection was confirmed by use of an ultrasound probe. The dura mater and arachnoid membrane were opened longitudinally. The $\mathrm{CH}$ was identified and completely dissected from the surrounding spinal cord. Myelotomy was not performed during the resection. Histopathological examination indicated $\mathrm{CH}$ (Fig. 2). The dura mater with arachnoid membrane was sutured with 5-0 nylon string and covered with a patch sprayed with fibrin glue, to prevent leakage of cerebrospinal fluid (CSF). An autologous bone strut from the iliac crest was grafted without hardware. The muscles were sutured, and the wound was closed. Transcranial electrical stimulation motor evoked potentials (MEPs) were used for intraoperative spinal cord monitoring, and no deterioration in their amplitude or latency was observed during the surgery. Estimated blood loss was $50 \mathrm{ml}$, and the operative time was 5 hours 23 minutes.

The postoperative course was uneventful, without CSF leakage, and the preoperative symptoms disappeared. Bony fusion was obtained six months postoperatively, and there has been no local recurrence two years after the surgery.

\section{Case 2}

A 28-year-old male presented with sudden right shoulder pain and motor weakness of the right upper extremity. MRI showed an intramedullary heterogeneous lesion at the ventral side of the $\mathrm{C} 6$ level, suggesting $\mathrm{CH}$ (Fig. 3). The surgical technique was similar to that used in Case 1, including tumor resection from an anterior approach and ACCF. Intraoperative spinal cord monitoring showed no deterioration in the MEP amplitude or latency. The estimated blood loss was $80 \mathrm{ml}$, and the operative time was 3 hours 40 minutes. Histopathological examination indicated $\mathrm{CH}$.

The postoperative course was uneventful, without CSF leakage. Bony fusion was obtained 6 months postoperatively, and no local recurrence was observed at the two-year follow-up (Fig. 4).

\section{Discussion}

Although a few studies have reported using the anterior 

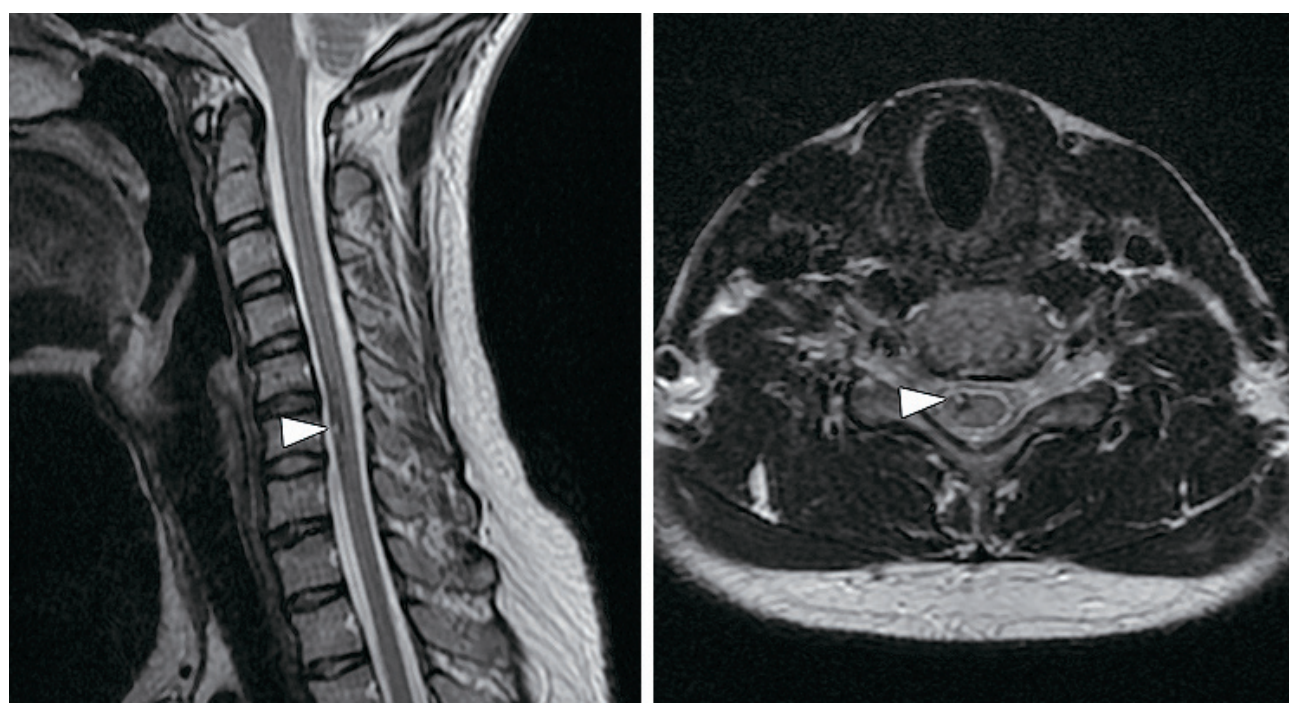

Figure 3. Preoperative T2-weighted MRI at sagittal (left) and axial (right) sections show an intramedullary heterogeneous lesion surrounded by a hypointense area at the ventral side of the C6 level (arrowheads).
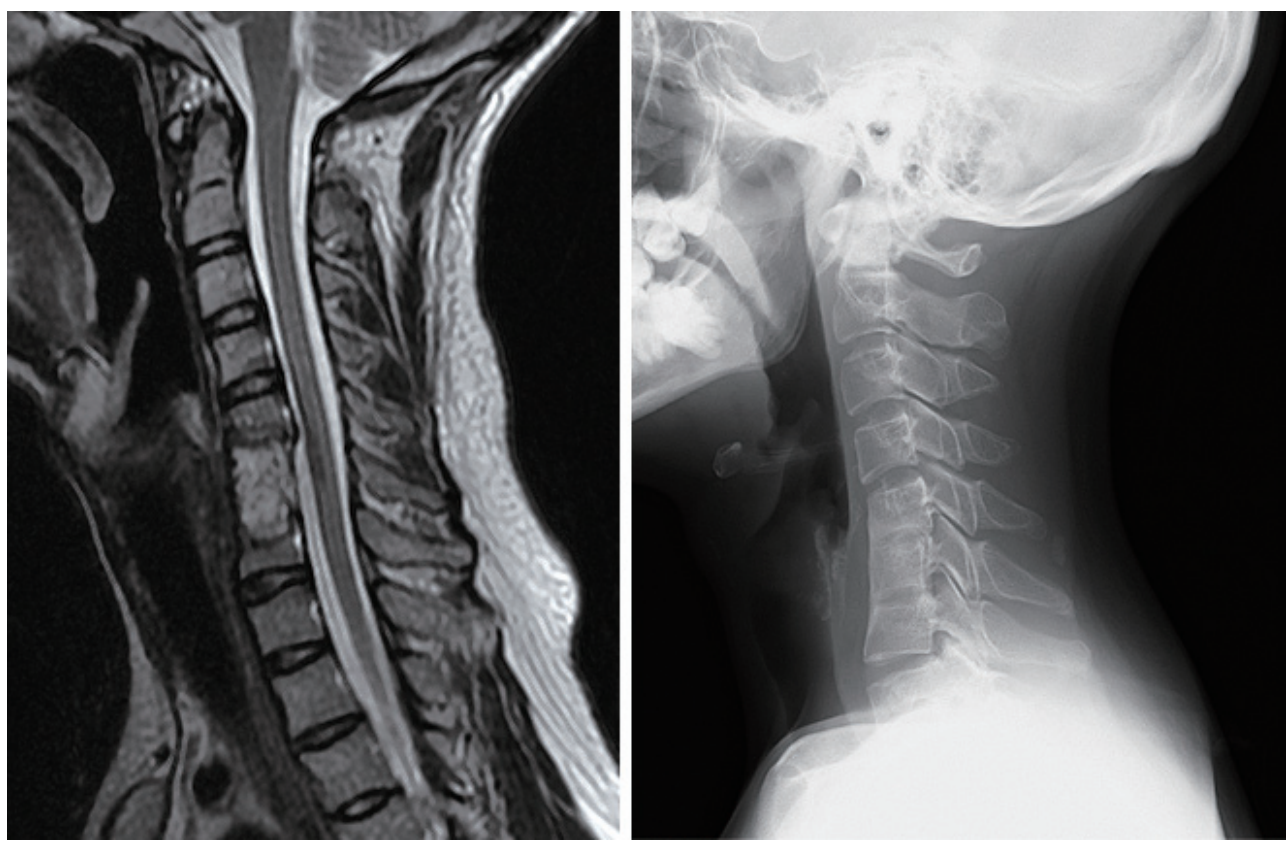

Figure 4. Postoperative MRI (left) and an X-ray image (right) show that the $\mathrm{CH}$ was resected and bony fusion has been achieved.

approach for $\mathrm{CH}$ resection at the cervical spinal cord, information is lacking about the detailed surgical technique or clinical outcomes at long-term follow-up ${ }^{3-6)}$. Here, we successfully resected intramedullary $\mathrm{CHs}$ on the cervical ventral side, as shown in our video images, and demonstrated stable outcomes without perioperative complications or recurrence at least two years after surgery. This approach makes it easier to directly resect a vascular malformation that exists at the ventral part of the spinal cord. Given the nature of a case report, however, further study is needed to validate the usefulness of this approach, by increasing the number of patients and observing them for a longer follow-up period.
A posterior approach is generally selected to access intramedullary sites. If the tumor or malformation exists on the ventral side, however, deep myelotomy is required, increasing the risk for postoperative neurological deterioration. Moreover, indirect access from the dorsal side to the ventral side can make it challenging to visualize the whole lesion, resulting in incomplete resection. Fontaine et al. advocated an anterolateral approach for ventral lesions ${ }^{7)}$, but this method carries a risk for vertebral artery injury.

The anterior approach has several disadvantages. Resection of the vertebral bodies with intervertebral discs and anterior and posterior longitudinal ligaments causes spinal in- 
stability, necessitating anterior fusion. In addition, the working space is limited, because of the deep and narrow surgical field. However, this approach is quite useful if the intramedullary lesion is small and appears on the ventral surface of the cervical spinal cord. This situation allows the surgeon to dissect the lesion without myelotomy, and this is a significant advantage of the anterior approach. In fact, several surgeons have used this approach for other intramedullary tumors, including hemangioblastomas, and reported that it is safe and effective, as in our cases ${ }^{8,9)}$. We conclude that this technique stands a good chance of achieving complete total resection, if the patients are strictly selected.

According to a previous report, residual $\mathrm{CH}$ observed on postoperative MRI often results in recurrence ${ }^{1)}$. Owing to the direct and total resection achieved in our cases, no residual lesions were detected on the postoperative images, suggesting a low possibility of local recurrence. However, longer follow-up observation is mandatory to confirm that the recurrence will not occur.

Conflicts of Interest: The authors declare that there are no relevant conflicts of interest.

Author Contributions: N.N, K.I, and M.N conceptualized this study. M.N conducted surgeries. K.K conducted pathological analysis. N.N, K.I, and M.N wrote original draft. N.N, K. I, K.K, O.T, E.O, N.F, M.Y, M.M, M.N, and K.W reviewed and edited the draft. K.I and M.N supervised this study.

\section{References}

1. Mitha AP, Turner JD, Abla AA, et al. Outcomes following resection of intramedullary spinal cord cavernous malformations: a 25year experience. J Neurosurg Spine. 2011;14(5):605-11.

2. Steiger HJ, Turowski B, Hanggi D. Prognostic factors for the outcome of surgical and conservative treatment of symptomatic spinal cord cavernous malformations: a review of a series of 20 patients. Neurosurg Focus. 2010;29(3):E13.

3. Reitz M, Burkhardt T, Vettorazzi E, et al. Intramedullary spinal cavernoma: clinical presentation, microsurgical approach, and long-term outcome in a cohort of 48 patients. Neurosurg Focus. 2015;39(2):E19.

4. Weil AG, Bhatia S. Resection of a ventral intramedullary cervical spinal cord cavernous malformation through an anterior approach. Neurosurg Focus. 2014;37 Suppl 2:Video 18.

5. Banczerowski P, Lipoth L, Vajda J, et al. Surgery of ventral intradural midline cervical spinal pathologies via anterior cervical approach: our experience. Ideggyogy Sz. 2003;56(3-4):115-8.

6. Nishikawa M, Ohata K, Ishibashi $\mathrm{K}$, et al. The anterolateral partial vertebrectomy approach for ventrally located cervical intramedullary cavernous angiomas. Neurosurgery. 2006;59(1 Suppl 1):ONS 58-63

7. Fontaine D, Lot G, George B. Intramedullary cavernous angioma. Resection by oblique corpectomy. Surg Neurol. 1999;51(4):43541.

8. Iwasaki Y, Koyanagi I, Hida K, et al. Anterior approach to intramedullary hemangioblastoma: case report. Neurosurgery. 1999;44 (3):655-7.

9. Imai $\mathrm{H}$, Ishii $\mathrm{K}$, Chikuda $\mathrm{H}$, et al. Successful surgical strategy for a cervical hemangioblastoma: case report. Surg Neurol Int. 2016;7 (Suppl 25):S660-3.

Spine Surgery and Related Research is an Open Access journal distributed under the Creative Commons Attribution-NonCommercial-NoDerivatives 4.0 International License. To view the details of this license, please visit (https://creativeco mmons.org/licenses/by-nc-nd/4.0/). 